\title{
Calibration of $\alpha$ Centauri and the Uniqueness of the Mixing Length
}

\author{
João Fernandes ${ }^{1,3}$, Corinne Neuforge ${ }^{2}$, Arlette Noels ${ }^{2}$ \\ and Annie Baglin ${ }^{1}$ \\ ${ }^{1}$ Département d'Astrophysique Stellaire et Galactique \\ Observatoire de Paris, Section de Meudon, France \\ ${ }^{2}$ Institut d'Astrophysique de l'Université de Liège, Belgium \\ ${ }^{3}$ Centro de Astrofísica da Universidade do Porto, Portugal
}

\begin{abstract}
Taking into account the uncertainties in the metallicity parameter, $Z$, we discuss the uniqueness of the mixing length convection parameter, $\alpha$, by calibration of the $\alpha$ Centauri binary system. Our results support the hypothesis of a unique value of $\alpha$ for both stars (equal to the solar one) for high values of $\mathrm{Z}$.
\end{abstract}

\section{Introduction}

The binary system $\alpha$ Cen A and B is, after the Sun, the stellar system where the observational quantities are known with highest accuracy.

- mass (Demarque et al., 1986; Heintz, 1982):

$$
M_{\mathrm{A}}=1.085 \pm 0.005 M_{\odot}, M_{\mathrm{B}}=0.900 \pm 0.01 M_{\odot}
$$

- absolute magnitude (Ochsenbein et al. 1984):

$$
M_{\text {bolA }}=4.317 \pm 0.038, M_{\text {bolB }}=5.546 \pm 0.038
$$

- effective temperature (Chmielewski et al., 1993):

$$
T_{\mathrm{effA}}=5800 \pm 20 K, T_{\mathrm{effB}}=5325 \pm 50 K
$$

The value of the metallicity parameter, $Z$, is still controversial. The most recent spectroscopic observations lead to slighty differents results: Meylan et al. 
(1992) give $\mathrm{Z} \simeq 0.031$ (a revision of the value obtained by Fürenlid \& Meylan, 1990: 0.026) whereas Chmielewski et al. (1993) give 0.035 .

\section{Calibrations}

Different methods of calibration can be proposed, depending on whether $Z$ is determined from observations or not.

\subsection{The case when $\mathrm{Z}$ is not available from observations}

Assuming knowledge of the masses and making the assumption of a unique mixing length convection parameter for both stars, the four observed quantities of the system, effective temperatures, $T_{\mathrm{effA}}, T_{\mathrm{efB}}$, and bolometric magnitudes $M_{\text {bolA }}, M_{\text {bolB }}$, allow a determination of the four unknown parameters, age t, $\alpha$, helium abundance $\mathrm{Y}$ and $\mathrm{Z}$. This calibration method is described in Noels et al. (1991). The solution is obtained by the resolution of their linear system (1). This system gives a "first order solution" which is adjustable to give the best fit between evolutionary tracks and observations. Computations have been made using both the Liège code (Noels et al., 1991) and the CESAM code (Morel et al., 1993). Our solution is:

$$
Y_{*}=0.320, Z_{*}=0.038, t_{*}=5.3 G y, \alpha_{*}=2.05
$$

In the next sections, this model will be refered as the "reference model". The value of $\alpha$ is close to the our calibrated solar one, 2.06, increasing confidence in our hypothesis.

\subsection{The case when $\mathrm{Z}$ is available from observations}

In this case the hypothesis of equal $\alpha$ for two stars is checked. $\mathrm{Z}$ is taken as fixed at the observational value and $\alpha_{\mathrm{A}} \neq \alpha_{\mathrm{B}}$ is considered. These calibrations proceed in two steps:

1) Using $\alpha=\alpha_{\text {SUN }}, t$ and $Y$ are determined so that calculated luminosities fit observed values at the same age;

2) Two convection mixing length parameters are then adjusted to reproduce the observed effective temperatures.

In the first aproximation, $M_{\text {bol }}$ is fixed by $\mathrm{Y}$ and $T_{\text {eff }}$ by $\alpha$. Calibrations performed at Yale (Demarque et al., 1986; Edmonds et al., 1992) use this method for a fixed $Z$-value: $Z=0.026$ (Fürenlid et al., 1990). Their solution is:

$$
Y_{*}=0.300, t_{*}=4.7 G y, \alpha_{\mathrm{A}}=1.15, \alpha_{\mathrm{B}}=1.25
$$

We also have performed this kind of calibration, using the interior opacities of Iglesias et al. (1992) and low-T opacities from Neuforge (1993). Our results are presented in Table 1. They are consistent with those of Lydon et al. (1993). 
Table 1

\begin{tabular}{lllll}
\hline $\mathrm{Z}$ & $\mathrm{t}$ & $\mathrm{Y}$ & $\alpha_{A}$ & $\alpha_{B}$ \\
\hline 0.040 & 5.61 & 0.324 & 2.10 & 2.09 \\
0.038 & 5.72 & 0.318 & 2.10 & 2.13 \\
0.030 & 5.93 & 0.296 & 2.03 & 2.18 \\
0.026 & 5.62 & 0.285 & 1.91 & 2.10 \\
0.020 & 5.10 & 0.267 & 1.74 & 2.03 \\
\hline
\end{tabular}

From Table 1 we see that as $Z$ increases, the mixing length convection parameters tend to the same value, close to the solar one, while for lower $\mathrm{Z}$, they are found to be different for both stars. This explains why Edmonds et al. (1992) find different values of $\alpha$.

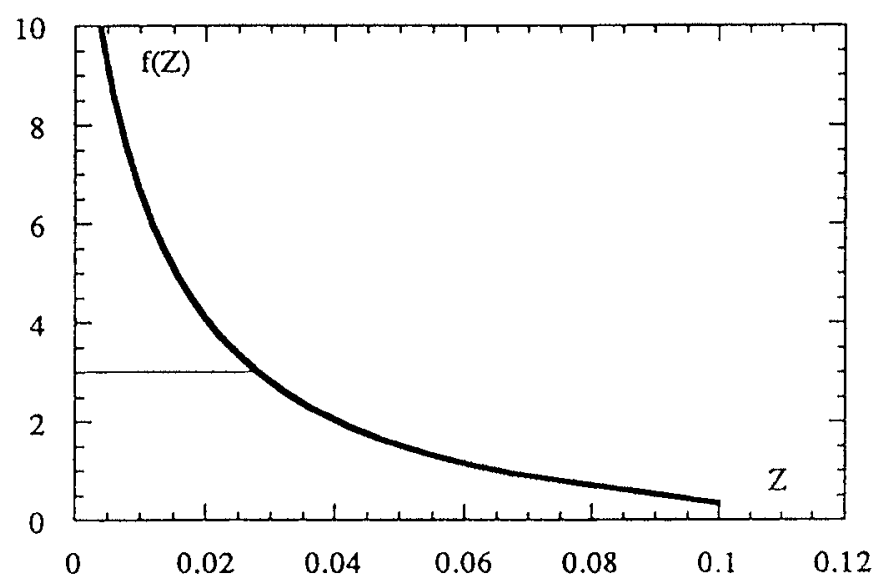

Fig. 1. $f(Z)=\frac{\Delta y}{\Delta Z}$ as function of $Z$

\section{Uniqueness of the solution}

Using homology theory, it is possible to show that for ZAMS models, there is a relation between $\mathrm{Y}$ and $\mathrm{Z}$. For a fixed mass and radius and Kramers law opacity, $K=K_{0} \rho T^{-3.5}$ ( $T$ temperature, $\rho$ density), the relation between the luminosity, $\mathrm{L}$, and mean molecular weight, $\mu$, is

$$
L \sim \frac{\mu^{7.5}}{K_{0}}
$$

Considering bound-free and free-free transitions as the main contributions to opacity, i.e., $K_{0} \sim K_{1} Z(2-Y-Z)+K_{2}(2-Y-Z)$ (Cox \& Giuli, 1968), we have, 


$$
\frac{\Delta L}{L}=\frac{\Delta M_{\mathrm{bol}}}{M_{\mathrm{bol}}}=0 \Rightarrow \frac{\Delta Y}{\Delta Z}=\mathrm{f}(\mathrm{Z})
$$

The variation of $f(Z)$ with $Z$ is illustrated in Figure 1. From this Figure we see that $f(Z)$ gets smaller with increasing $Z$. This means that, considering two ZAMS models at high $Z$-values, if the ratio of their $Y$ and $Z$ difference, i. e, $f(Z)$, is lower than 3 , for instance, the models will have aproximately the same $M_{\mathrm{bol}}$.

We have constructed $\alpha$ Cen A and B models (Figure 2 and 3) for metallicity values of 0.035 and 0.043 , where the $\mathrm{Y}$ and $\mathrm{Z}$ values satisfy the relation,

$$
\frac{\Delta Y}{\Delta Z}=\frac{Y-Y_{*}}{Z-Z_{*}}=2.74
$$

where "*", means the "reference model" $\left(Z_{*}=0.038\right)$.

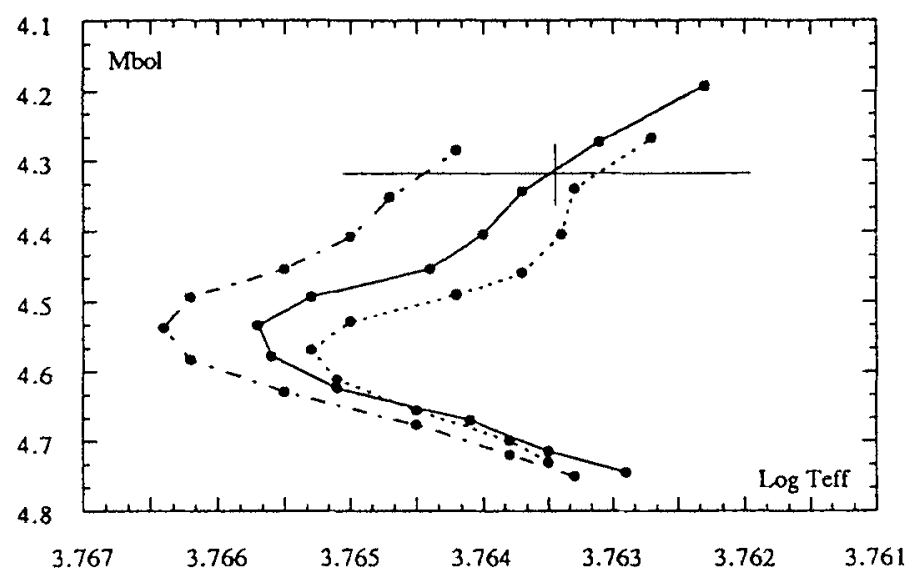

Fig. 2. $\alpha$ Cen A evolutionary tracks for $Z=0.035$ (dot-dashed line), 0.043 (short-dashed line, and 0.038 (solid line-"reference model"). Dots gives the model evolution, from ZAMS to $5.3 \mathrm{~Gy}$ by steps of $0.5 \mathrm{~Gy}$. The errors in the observations are shown

From these figures we can infer that, taking into account the errors in the observations, we cannot distinguish (especially in $M_{\text {bol }}$ ) between these three calibrations, which is the best. Only more accurate values of $\mathrm{Z}$ would allow it. These results provide a numerical confirmation for the variation of $f(Z)$.

Even so, we have checked that it is not possible to have the same HR position for two models when only variations $(Y, Z)$ are made, the determinant of the linear system (1) from Noels et al. (1991) being nonzero. This supports the validity of the calibration method presented in 2.1 . 


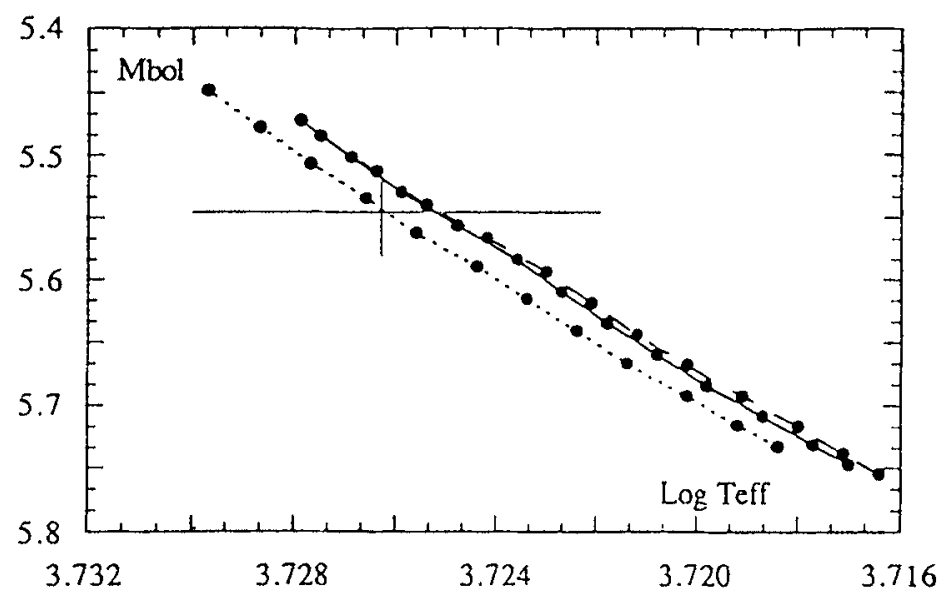

Fig. 3. $\alpha$ Cen B evolutionary tracks for $Z=0.035$ (long-dashed line), 0.043 (shortdashed line, and 0.038 (solid line-"reference model"). Dots gives the model evolution, from ZAMS to 6 Gy by steps of $0.5 \mathrm{~Gy}$. The errors in the observations are shown

\section{Conclusion}

These results provide us with a test for the uniqueness of the mixing length convection parameter since a high observational value for $Z$, i. e. $>0.030$, favours the hypothesis of a unique $\alpha$. On the other hand, because of the existence of the observational errors, a unique calibration for high Z-values is more difficult.

\section{Acknowledgments}

JF acknowledges the award of a scholarship from Junta Nacional de Investigação Cientifica e Tecnológica through "Programa Ciência", from Portugal.

\section{References}

Chmielewski, Y., Friel, E., Cayrel de Strobel, G., Bentolila, C. (1992): Astron. Astrophys., 263, 219

Cox, J.P., \& Giuli R.T. (1968): "Stellar Structure - Physical Principles", Vol.I, Eds. Gordon and Breach

Demarque, P., Guenther, D.B., Van Altena, W.F. (1986): Astrophys. J., 300, 773

Edmonds, I., Lawrence, C., Demarque, P., Guenther, D.B., Pinsonneault, H.M. (1992): Astrophys. J., 394, 313

Fürenlid, I., \& Meylan, T. (1990): Astrophys. J., 350, 827

Heintz W.D. (1982): Observatory, 102, 42

Iglesias, C.A., Rogers, F.J., Wilson, B.J. (1992): Astrophys. J., 397, 717 
Lydon, T.J., Fox, P.A., Sofia, S. (1993): in press

Meylan, T., Meylan, P. \& Fürenlid, I. (1992): BAAS, 23, 1384

Morel, P. (1992): "Inside the stars",445, Proceedings of IAU coll. 137. Eds. W. Weiss and $A$. Baglin

Neuforge C. (1993): Astron. Astrophys., 274, 818

Noels, A., Grevesse, N., Magin, P., Neuforge, C., Baglin, A., Lebreton, Y. (1991): Astron. Astrophys., 247, 91

Ochsenbein F., Acker A., Legrand E., Poncelet J.M., Thuet-Fleck E. (1984): "Catalogue des étoiles plus brillantes", eds. A. Acker, Obs. Strasbourg, Centre de Données Stellaires et Planétarium 